\title{
CCL28 Gene
}

National Cancer Institute

\section{Source}

National Cancer Institute. CCL28 Gene. NCI Thesaurus. Code C99630.

This gene is involved in chemotaxis. 